\title{
Kurzanalysen
}

Hannes Adomeit*

\section{Russland im Strategischen Konzept der NATO}

https://doi.org/10.1515/sirius-2021-4008

\section{Einleitung}

Auf ihrem Treffen im Juni 2021 in Brüssel haben die Staatsund Regierungschefs der NATO offiziell die Erstellung eines neuen Strategischen Konzepts beauftragt, das beim nächsten NATO-Gipfel 2022 in Spanien vorgestellt werden soll. Das letzte Strategische Konzept stammt aus dem Jahr 2010 und gilt mit Blick auf die geo- und sicherheitspolitischen Umbrüche als überholt, insbesondere weil sich die Beziehungen zu Russland grundsätzlich verändert haben und von Russland eine militärische Bedrohung für Mitgliedstaaten des Bündnisses ausgeht. Auf der Gipfelkonferenz im Dezember 2019 in London hatte die NATO auf deutsche Initiative einen sogenannten Reflexionsprozess auf den Weg gebracht. In den Monaten darauf erarbeitete eine Expertengruppe um Bundesminister a. D. Thomas de Maizière Empfehlungen für mögliche Reformen der NATO, die auch den Umgang mit Russland betrafen. Aufbauend auf den Vorschlägen legte NATO-Generalsekretär Jens Stoltenberg eine ambitionierte Agenda NATO 2030 vor, die zusammen mit dem Reflexionspapier im Mittelpunkt der Gespräche auf dem Gipfel in Brüssel stand.

Das Strategische Konzept wird im Bündnis als das zentrale strategische Grundlagenpapier betrachtet, das die wichtigsten Herausforderungen beschreibt, denen sich die NATO gegenübersieht, und die notwendigen Maßnahmen umreißt, um ihnen zu begegnen. Seit Ende des Kalten Krieges wurden drei Strategische Konzepte verfasst, 1991, 1999 und 2010. In allen spielt das Verhältnis zu Russland eine wichtige, im ersten und letzten Konzept eine zentrale Rolle. Wie ein roter Faden durchziehen diese Dokumente Erwartungen und Hoffnungen, das neue Russland möge seine imperialen Groß- und Weltmachtambitionen nachhaltig aufgeben und sich zu einem Staat wandeln, der sich europäischen Werten verpflichtet sieht. Russlands Annexion der Krim und seine Militärintervention in der Ostukraine 2014 markierten einen Wendepunkt, der die

*Kontakt: Dr. Hannes Adomeit, Senior Fellow, Institut für Sicherheitspolitik an der Universität Kiel, E-Mail: hannes-adomeit@t-online.de jahrzehntelangen Hoffnungen und auf ihnen beruhenden Annahmen grundlegend in Frage gestellt hat. Im Folgenden werden die in den drei Konzepten diagnostizierten, von Russland ausgehenden Herausforderungen und die resultierenden Maßnahmen dargelegt und ihre jeweilige Bedeutung für die Entwicklung der NATO-Russland-Beziehungen herauskristallisiert. In diesem Kontext sollen Schwächen der bisherigen Strategiedokumente, sofern sie sich mit Russland befassten, aufgezeigt und der Revisionsbedarf hinsichtlich des Strategischen Konzepts 2022 formuliert werden.

\section{Das Strategische Konzept 1991}

Die Sterne für eine tiefgreifende und umfassende Neuordnung des Verhältnisses zwischen der NATO, dem wiedervereinigten Deutschland und dem neuen Russland nach dem Zusammenbruch der Sowjetunion standen günstig. Die Außenpolitik der Russischen Föderation ab ihrer Souveränitätserklärung (Juni 1990) mit Andrej Kozyrev an der Spitze des entsprechenden Ministeriums (ab Oktober 1990) beruhte auf dem in der Gorbatschow-Ära entwickelten Konzept des Neuen Politischen Denkens. Dazu gehörte der Abbau des Feindbilds Westen, der angeblich immer und ewig darauf aus sei, Russland in die Knie zu zwingen.

Das Strategische Konzept vom November 1991 trug dieser Entwicklung Rechnung. Es stellte fest, dass „seit 1989 in Mittel- und Osteuropa tiefgreifende politische Veränderungen stattgefunden haben, die das Sicherheitsumfeld, in dem die NATO ihre Ziele zu erreichen versucht, radikal verbessert haben. Die ehemaligen Satelliten der UdSSR haben ihre Souveränität vollständig zurückerlangt. Die Sowjetunion und ihre Republiken befinden sich im Umbruch. Die drei baltischen Republiken haben ihre Unabhängigkeit wiedererlangt. Sowjetische Truppen haben Ungarn und die Tschechoslowakei verlassen und sollen ihren Rückzug aus Polen und Deutschland bis 1994 vollenden. Die Länder, die früher NATO-Gegner waren, haben den Warschauer Pakt zerlegt und ideologische Feindschaft zum Westen aufgegeben. Sie haben in unterschiedlichem Maße politische Maßnahmen ergriffen und mit deren Umsetzung begonnen, die auf pluralistische Demokratie, Rechtsstaatlichkeit, Achtung der Menschenrechte 
und Marktwirtschaft abzielen. Die politische Spaltung Europas, die die militärische Konfrontation in der Zeit des Kalten Krieges auslöste, ist damit überwunden.“1

Die Politik des neuen Russland fügte sich in dieses Bild ein. So erklärte Präsident Boris Jelzin Ende 1991 im Sicherheitsrat der Vereinten Nationen und anschließend bei einem Besuch in Kanada, Russland betrachte die westlichen Länder nicht lediglich als Partner, sondern als „Verbündete.“2 Dieser Logik entsprechend erklärte er die Mitgliedschaft des Landes in der NATO zu einem langfristigen Ziel. ${ }^{3}$ Zudem bekannte er sich zu gemeinsamen Werten. Die Prinzipien, auf denen Russlands Politik beruhe, seien klar und einfach: „Vorrang der Demokratie, Menschenrechte und Freiheiten, rechtliche und moralische Standards. “4

Das Strategische Konzept enthielt sich trotz aller konstatierten positiven Entwicklungen jeglicher Euphorie. Das Ende der Ost-West-Konfrontation habe das Risiko eines schweren Konflikts in Europa stark verringert, er sei aber nicht endgültig auszuschließen. Selbst in einem kooperativen Verhältnis blieben die militärischen Fähigkeiten und das Aufbaupotenzial der Sowjetunion [sic], einschließlich ihrer nuklearen Dimension, immer noch der wichtigste Faktor, den das Bündnis bei der Wahrung des strategischen Gleichgewichts in Europa bedenken müsse. Zudem dürfe eine Bewertung der mit dem Veränderungsprozess verbundenen Risiken und Unsicherheiten nicht außer Acht lassen, dass die Sowjetunion über deutlich stärkere konventionelle Streitkräfte verfüge als jeder andere europäische Staat und über ein Nukleararsenal, das nur dem der USA vergleichbar sei. Man müsse das tatsächliche militärische Potenzial der Sowjetunion berücksichtigen, um Stabilität und Sicherheit in Europa zu erhalten. ${ }^{5}$

Diese Besonnenheit des Konzepts erwies sich bereits nach zwei Jahren als berechtigt. Das „neue“ Russland kehrte zu wesentlichen Aspekten des Alten Denkens

1 Strategisches Konzept 1991, I, 1.

2 „Russland betrachtet die USA und den Westen nicht als bloße Partner, sondern als Verbündete“, so Jelzin am 20. Januar 1992 im UN Sicherheitsrat. Text of Boris Yeltsin's Speech to the United Nations Security Council, AP News, 31.1.1992. Ähnlich äußerte er sich am 2. Februar 1992 bei seiner Ankunft in Ottawa, siehe Michael Wines: Bush and Yeltsin Declare Formal End to Cold War, New York Times, 2.2.1992, A 1.

3 Offiziell in einem Brief an die NATO. Dort hieß es: „Heute stellen wir die Frage der Mitgliedschaft Russlands in der NATO, betrachten sie jedoch als langfristiges politisches Ziel.“ Zitiert nach Thomas L. Friedman: Soviet Disarray; Yeltsin Says Russia Seeks to Join NATO, New York Times, 21.12.1991, A 5.

4 Jelzin am 20.1.1992 im UN Sicherheitsrat, siehe Fn 2.

5 Strategisches Konzept 1991, I, 10. Das Strategische Konzept 1991 spricht rein rechtlich gesehen ganz richtig noch von der Sowjetunion. Offiziell endete ihre Existenz erst am 31. Dezember 1991. zurück. Das machte hinsichtlich der NATO eine Studie des russischen Auslandsgeheimdienstes (SVR) unter Leitung Jewgeni Primakows deutlich. Das im November 1993 vorgestellte Dokument bezeichnete die NATO erneut als die „größte militärische Gruppierung der Welt, die ein enormes Offensivpotenzial besitzt.“ Es unterstellte der westlichen Militärallianz, „den Stereotypen des Blockdenkens" verhaftet $\mathrm{zu}$ sein, und warnte sie eindringlich vor einer Ausdehnung nach Osten. ${ }^{6}$ Jelzins Pressesprecher mahnte sogar, ein Ausbau der NATO in Gebiete unmittelbar an der russischen Grenze würde zu einer „militärpolitischen Destabilisierung der Region“ führen. ${ }^{7}$

\section{Das Strategische Konzept 1999}

Trotz des zunehmend schwierigen Verhältnisses zwischen dem Westen und dem Russland Jelzins setzte das im April 1999 in Washington verabschiedete Strategische Konzept einen deutlich positiven Akzent. ${ }^{8}$ So heißt es dort: „Es entsteht ein neues, stärker integriertes Europa und eine euro-atlantische Sicherheitsstruktur, in der die NATO eine zentrale Rolle spielt. Das Bündnis steht im Mittelpunkt der Bemühungen um die Etablierung neuer Muster der Zusammenarbeit und des gegenseitigen Verständnisses in der euro-atlantischen Region und hat sich im Interesse einer breiteren Stabilität zu wesentlichen neuen Aktivitäten verpflichtet. “9

Wie aber fügte sich Russland in diese neue Sicherheitsstruktur ein, die der NATO eine zentrale Rolle zuwies? Im Wesentlichen problemlos, denn „im Rahmen der NATO-Russland-Grundakte über gegenseitige Beziehungen, Zusammenarbeit und Sicherheit haben sich die NATO und Russland verpflichtet, ihre Beziehungen auf der Grundlage von gemeinsamem Interesse, Gegenseitigkeit und Transparenz zu entwickeln, um einen dauerhaften

6 Die Studie hatte den Titel Služba vneshnej razvedki und erschien 1993. Der 30 Seiten umfassende Text der Studie wurde an Journalisten auf einer Pressekonferenz in Moskau verteilt und vollständig oder in Auszügen in allen großen russischen Zeitungen veröffentlicht. Primakov löste Kozyrev im Januar 2006 in seinem Amt als Außenminister ab und war von September 1998 bis Mai 1999 Premierminister.

7 ITAR-TASS (russ.), 5.1.1994.

8 Die im Strategischen Konzept 1999 gezeigte positive und optimistische Sicht des gegenwärtigen Stands und künftiger Möglichkeiten der Beziehungen zu Russland fand ihre Entsprechung in der Gemeinsamen Strategie der EU gegenüber Russland vom Juni 1999. Dort wurde die „strategische Partnerschaft“ mit Russland nicht als entfernte Vision, sondern als bereits bestehende Wirklichkeit behandelt, siehe Europäischer Rat 1999.

9 Strategisches Konzept 1999, 1. 
und integrativen Frieden im euroatlantischen Raum zu erreichen, der auf den Grundsätzen der Demokratie und der kooperativen Sicherheit basiert. Die NATO und Russland haben vereinbart, ihrem gemeinsamen Engagement für den Aufbau eines stabilen, friedlichen und ungeteilten Europas konkrete Bedeutung zu verleihen.“10

Die Glaubwürdigkeit derartiger Vereinbarungen und Zusicherungen hinterfragte man nicht. Folgerichtig sah man auch die Osterweiterung der NATO nicht als ernstes Problem in den Beziehungen zu Russland an. „Das Bündnis bleibt gemäß Artikel 10 des Washingtoner Vertrags für neue Mitglieder offen [und] erwartet, in den kommenden Jahren weitere Einladungen an Nationen auszusprechen, die bereit und in der Lage sind, die Verantwortung und Verpflichtungen einer Mitgliedschaft zu übernehmen." $" 11$

Eine Mitgliedschaft der Ukraine in der NATO wurde nicht ausdrücklich befürwortet, aber auch nicht ausgeschlossen. Zudem zeigte sich die NATO „entschlossen, ihre besondere Partnerschaft mit der Ukraine auf der Grundlage der NATO-Ukraine-Charta weiter zu stärken, einschließlich politischer Konsultationen zu Fragen gemeinsamen Interesses und einer breiten Palette praktischer Kooperationsaktivitäten, [und] weiterhin die Souveränität und Unabhängigkeit der Ukraine, die territoriale Integrität, die demokratische Entwicklung, den wirtschaftlichen Wohlstand und ihren Status als Nichtnuklearwaffenstaat als Schlüsselfaktoren für Stabilität und Sicherheit in Mittel- und Osteuropa und in Europa insgesamt zu unterstützen. “12

Am 24. März 1999 begann die NATO mit der Bombardierung Serbiens. Das Konzept erwähnte nur mit wenigen Worten diesen Schritt, der über das offizielle Russland in der Endphase der Jelzin-Ära hinaus zu einem Aufschrei der Empörung in der russischen Öffentlichkeit führte und das Verhältnis zu Russland ernsthaft und nachhaltig belastete. Es lobte die Bemühungen der NATO, dem „immensen menschlichen Leid, das durch die Konflikte auf dem Balkan verursacht wurde“, ein Ende zu setzen und bezeichnete das Engagement des Bündnisses als „,beispielhaft für Konfliktprävention und Krisenbewältigung.“13

Zum Zeitpunkt der Veröffentlichung des Strategischen Konzepts war Putin Chef des Inlandsgeheimdiensts FSB und Sekretär des Sicherheitsrates. Sein meteorhafter Aufstieg zum Präsidentenamt, der mit dem Zweiten Tschetschenienkrieg einherging, und die von ihm in die Wege

10 Strategisches Konzept 1999, 36 11 Strategisches Konzept 1999, 39. 12 Strategisches Konzept 1999, 37. 13 Strategisches Konzept 1999, 12. geleitete Abkehr von einem auf Demokratie und Rechtsstaat im Inneren und imperialen und „Großmacht“-Ambitionen nach außen gerichteten Weg hin zu Autoritarismus und Konfrontation mit dem Westen waren nicht vorhersehbar, wie das Strategische Konzept 1999 bezeugt. Mangelnde Voraussicht und eine ungenügende wirklichkeitsgetreue Bestandsaufnahme kennzeichneten ebenfalls das Strategische Konzept, das nach rund einem Jahrzehnt in Lissabon im November 2010 verabschiedet wurde.

\section{Das Strategische Konzept 2010}

In puncto Bestandsaufnahme und Umgang mit Russland enttäuscht das Konzept von 2010 auf ganzer Linie. In seiner Beschreibung des Sicherheitsumfelds befindet sich „der euro-atlantische Raum in Frieden“. Wiederum ohne den tatsächlichen Charakter des Verhältnisses zu Putins Russland zu hinterfragen, stellt es lapidar fest: „Die Beziehungen zwischen der NATO und Russland basieren auf den Zielen, Grundsätzen und Verpflichtungen der NATO-Russland-Grundakte und der Erklärung von Rom, insbesondere hinsichtlich der Achtung demokratischer Grundsätze und der Souveränität, Unabhängigkeit und territorialen Integrität aller Staaten im euro-atlantischen Raum.“ In einem Halbsatz werden „unterschiedliche“ Auffassungen zu „bestimmten Fragen“ erwähnt. Die NATO bliebe jedoch „davon überzeugt, dass die Sicherheit der NATO und Russlands eng verflochten ist und dass eine starke und konstruktive Partnerschaft, die auf gegenseitigem Vertrauen, Transparenz und Vorhersehbarkeit beruht, unserer Sicherheit am besten dienen kann. [Infolgedessen sei das Bündnis] entschlossen, die politischen Konsultationen und die praktische Zusammenarbeit mit Russland in Bereichen von gemeinsamem Interesse, einschließlich Raketenabwehr, Terrorismusbekämpfung, Drogenbekämpfung, Bekämpfung der Piraterie und Förderung einer breiteren internationalen Sicherheit, zu verstärken [und] das volle Potenzial des NATO-Russland-Rates für Dialog und gemeinsames Handeln mit Russland nutzen. "14

2010 konnte von der „Achtung demokratischer Grundsätze“ des bereits zu diesem Zeitpunkt fest verankerten Systems Putin keine Rede mehr sein - ein Fakt, den man aufgrund der auch oder gerade für Russland geltenden engen Verbindung zwischen Innen- und Außenpolitik hätte in Rechnung stellen müssen. ${ }^{15}$

14 Strategisches Konzept 2010, 33-34.

15 Der Zusammenhang zwischen russischer Innen- und Außenpolitik unter Putin ausführlich bei Adomeit 2017a und 2017b. 
Dieses Manko trifft auch auf die „Achtung der Souveränität, Unabhängigkeit und territorialen Integrität“ in Europa zu. Als Beispiel für die eklatante Verletzung dieser Prinzipien weist das Dokument nicht einmal andeutungsweise auf die russische Militärintervention in Georgien im August 2008 hin, auf die danach erfolgte Schaffung der Separatistenrepubliken Abchasien und Südossetien, auf deren Anerkennung durch Moskau und die dauerhafte Stationierung russischer Truppen in diesen abgetrennten Gebieten.

Gleichfalls ausgeklammert blieb die Problematik der Osterweiterung der NATO. Das Dokument erwähnt knapp, dieser Schritt habe „wesentlich zur Sicherheit der Verbündeten beigetragen“ und die „Tür zur NATO-Mitgliedschaft" bleibe für alle europäischen Demokratien offen. Der scharfe Widerstand Russlands, den Putin auf und kurz nach der Sitzung des NATO-Russland-Rats in Bukarest im April 2008 geäußert hatte, fiel unter den Tisch. ${ }^{16}$

Nur zwischen den Zeilen lassen sich einige der unterschiedlichen Auffassungen bei bestimmten Themen herauslesen. Dazu gehörten (1) die Raketenabwehr in Europa:

16 Offensichtlich mit Blick auf die Ukraine und Georgien erklärte Putin: ,Wir betrachten das Erscheinen eines mächtigen Militärblocks an unseren Grenzen, dessen Mitglieder sich nach Artikel 5 des Washingtoner Vertrags richten, als direkte Bedrohung der Sicherheit unseres Landes“, Vladimir Putin: Press Statement and Answers to Journalists' Questions Following a Meeting of the Russia-NATO Council, Kremlin.ru, 4.4.2008, http://en.kremlin.ru/events/president/ transcripts/24903. Zwei Tage später in Sotschi gegenüber Präsident George W. Bush erklärte er, dass „die Ukraine kein echter Staat“ sei. Ein Großteil ihres Territoriums sei von Russland „verschenkt“ worden. Die Westukraine möge zu Osteuropa gehören, die Ostukraine sei aber „unser“ - russisches - Gebiet, und sollte die Ukraine der NATO beitreten, könnte Russland die Ostukraine übernehmen und die Ukraine praktisch „aufhören, als Staat zu existieren“. Er stellte auch die territoriale Integrität Georgiens in Frage. Den kommenden Schritt des Kremls vorwegnehmend warnte er Bush, Russland werde, sollte Georgien sich weiter auf eine NATO-Mitgliedschaft zubewegen, Abchasien und Südossetien anerkennen. Siehe Socor 2008, Blank 2008. Siehe auch den Bericht „Moskau warnt vor NATO-Aufnahme Georgiens und der Ukraine“, Spiegel online, 8.3.2008. Die Anfechtung der Souveränität und Legitimität der Ukraine ist im System Putin fest verankert und hat im Juli 2021 in einem Artikel des Präsidenten „Über die historische Einheit von Russen und Ukrainer“ ihren vorläufigen Höhepunkt gefunden. Die Ukraine, so Putin, werde als „gegen Russland gerichtete Plattform“ genutzt. Dem liege die Absicht zugrunde, die Ukraine „direkt von außen zu kontrollieren, die ukrainischen Behörden, Sicherheitsdienste und Streitkräfte durch ausländische Berater zu überwachen, die militärische Entwicklung des Territoriums der Ukraine [voranzutreiben] und der NATO Infrastruktur bereitzustellen“, siehe Vladimir Putin: Stat'ja Vladimira Putina “Ob istoričeskom edinstve russkich i ukraincev” [Article by Vladimir Putin "On the Historical Unity of Russians and Ukrainians”], Kremlin.ru, 12.07.2021, http://kremlin.ru/events/president/news/66181. „die Entwicklung von Fähigkeiten [der NATO], unsere Bevölkerungen und Territorien gegen ballistische Raketenangriffe zu verteidigen“; (2) die in Europa stationierten Nuklearwaffen: deren höhere Anzahl im russischen Arsenal und große Nähe zum Gebiet der NATO-Mitglieder sowie mangelnde Transparenz seitens Russland; (3) die Disparität zwischen Russland und der NATO bei nuklearen Kurzstreckenwaffen. ${ }^{17}$

Wie ist dieser milde Blick so kurz nach dem historischen Tief der Beziehungen zwischen Russland und der NATO nach der russischen Militärintervention in Georgien zu erklären? ${ }^{18} 2009$ wurde Barack Obama als US-amerikanischer Präsident vereidigt und seine Regierung bemühte sich um einen „Neustart“ (reset) der russisch-amerikanischen Beziehungen. Bis Ende 2011 ließ sich Washingtons Ansatz durchaus als erfolgreich bezeichnen. Grund war hauptsächlich die Rochade der Ämter von Präsident (Putin) und Premier (Dmitri Medwedew) im Jahr 2008 und Russlands Politik in dieser „Tandem“-Periode bis 2012.

Der neue Präsident Medwedew erklärte grundlegende wirtschaftliche und gesellschaftliche Reformen zur „Überlebensfrage“ für Russland und leitete eine Modernisierungskampagne ein. ${ }^{19}$ Die Modelle und Partner waren klar benannt und eindeutig im Westen zu finden. Im Juni 2009 besuchte Medwedew die USA und machte sich nach seiner Rückkehr für den Aufbau eines Innovationszentrums in Skolkowo nahe Moskau stark, das sich am Modell Silicon Valley ausrichten sollte. Europa sollte zur Modernisierung von Wirtschaft und Gesellschaft beitragen. Im Juni 2010 stimmte Moskau neuen Sanktionen gegen Iran zu, um Teheran von der Entwicklung von Atomwaffen abzuhalten. Ein neuer Vertrag über die Reduktion strategischer Waffen (START) wurde ausgearbeitet und von der Duma im Januar 2011 ratifiziert. Im März 2011 enthielt sich Moskau bei der Abstimmung im UN-Sicherheitsrat über einen Militäreinsatz gegen Libyen. Medwedews Kampagne zog auch die Beziehungen zur EU ein, unter anderem mit der Unterzeichnung einer EU-Russland-Modernisierungs-

17 Strategisches Konzept 2010, 19, 26, 34. Die Nummerierung ist die des Autors, nicht des NATO-Dokuments.

18 Verschiedene Teilnehmer an der NATO-Russland-Ratstagung in Lissabon, berichtete der regierungsunabhängige russische Militärexperte Alexander Golts, hätten die Tagung als „historischen Durchbruch" in den NATO-Russland-Beziehungen bezeichnet. Insgesamt hätte er diesen Begriff zehn Mal gehört, siehe Alexander Golts: NATO Celebration Misses the Point, Moscow Times, 23.11.2010.

19 Zur Entwicklung in Russland von sozio-ökonomischer Modernisierung und Kooperation mit dem Westen à la Medwedew hin zu national-patriotischer Mobilisierung und Konfrontation mit dem Westen unter Putin siehe Adomeit 2017a, 42-45. 
partnerschaft im Mai 2010. Der Kreml bemühte sich ferner um eine Verbesserung seines Verhältnisses zu Polen. Zusammen mit seinem polnischen Amtskollegen Donald Tusk besuchte Medwedew als erster russischer oder sowjetischer Regierungschef im April 2010 die Gedenkstätte von Katyn und legte am Mahnmal für Tausende vom sowjetischen Geheimdienst NKWD ermordete polnische Offiziere und Intellektuelle einen Kranz nieder. Schließlich schien sich auch die russische Politik im postsowjetischen Raum geändert $\mathrm{zu}$ haben. Laut Außenminister Lawrow sollte die „Modernisierungsstrategie“ unter anderem in den Nachbarstaaten die Wahrnehmungen verändern und ein attraktiveres Bild von Russland vermitteln. Russland sei also, um es mit einer neuen politikwissenschaftlichen Floskel auszudrücken, mit größerer Soft Power auszustatten. $^{20}$

Zusammenfassend erscheint die NATO im Sicherheitskonzept 2010 „weniger als Verteidigungsorganisation, sondern vor allem als Krisenmanagerin jenseits der Bündnisgrenzen, als Partnerschaftsagentur sowohl Russland gegenüber als auch bei der weltweiten Sicherheitskooperation sowie als politische Transformationshelferin für Beitrittsaspiranten in Südosteuropa. Bündnisverteidigung gemäß Artikel 5 des Washingtoner Vertrags blieb zwar nominell Existenzgrundlage der Allianz, galt jedoch kaum als realistisches Szenario."21 Bereits Ende des folgenden Jahres wurde dieses Selbstverständnis der NATO grundlegend infrage gestellt.

\section{Grundlegend neuer Kontext}

Der Wendepunkt zu einem fundamental veränderten Kontext ist in Russland zu finden, in den Wahlen zur Duma am 4. Dezember 2011 und zum Präsidentenamt am 4. März 2012. ${ }^{22}$ Bei der Wahl zum Parlament erlitt die Kreml-nahe Partei Einheit Russlands schwere Verluste und Putins Ergebnisse blieben gleichfalls hinter den Werten von 2004 zurück. Folgenschwer waren in beiden Fällen allerdings nicht die Wahlergebnisse, sondern die Massendemonstrationen gegen Wahlfälschungen und Wahlmanipulationen. Zehntausende von Menschen gingen auf die Straße, bezeichneten die Regierungspartei als ein Sammelbecken

20 Russia Does not Want „Zero-Sum“ Geopolitical Games in CIS, Außenminister Lavrow in Westi TV, 10.12.2008.

21 Kamp 2017, 26-27.

22 Die Wende und ihre Konsequenzen ausführlich bei Adomeit 2017a, 44-49. von „Dieben und Gaunern“ und forderten ein „Russland ohne Putin.“23

Der Kreml zog aus den Ereignissen den naheliegenden Schluss: Der angeblich von westlichen Geheimdiensten und „sogenannten“ Nichtregierungsorganisationen nach Ostmitteleuropa eingeschleppte Virus der „Farbrevolutionen“ hatte begonnen, Russland zu infizieren. Aus Sicht der Moskauer Machtelite musste man dieser Entwicklung im Land selbst und in den Nachbarstaaten energisch Einhalt bieten. In der Innenpolitik sind seitdem repressive Maßnahmen getroffen worden, um pro-westliche - demokratische, liberale, rechtsstaatliche und autonome zivilgesellschaftliche - Bewegungen und Organisationen auszuschalten. Dies hat der Kreml mit einer anti-westlichen, national-patriotischen Mobilisierung verbunden.

In seiner europäischen Nachbarschaft fand dies in der Annexion der Krim und dem Krieg in der Ostukraine seinen Ausdruck, gegenüber dem Westen in der wieder geäußerten prinzipiellen Gegnerschaft. ${ }^{24}$ Diese ist keineswegs lediglich theoretisch und verbal, sondern sie ist materiell. Sie ist kein loses, auf ein inhaltlich und zeitlich begrenztes Ziel ausgerichtetes Maßnahmenbündel, kein „Zufallsprodukt [sondern] eine Strategie der hybriden Kriegsführung.“ ${ }^{25}$ Der Kreml setzt diese hybride Kriegsführung nicht allein „in Form von Cyber-Desorientierung und Desinformation“ ${ }^{26}$ und mit anderen nicht-militärischen Mitteln ein, sondern auch unter Anwendung oder Androhung sowohl konventioneller wie nuklearer militärischer Machtinstrumente. Letzteres geschieht in Russlands unmittelbarer Nachbarschaft und auch weit über seine Landesgrenzen hinaus. Es kann in Form des direkten Truppeneinsatzes erfolgen, wie in Georgien 2008, auf der Krim und in der Ostukraine 2014 und in Syrien nach 2015. Genauso kann es jedoch zu unprovozierten Truppenaufmärschen und umfangreichen Militärmanövern führen, bei denen -wie im April 2021 - unklar ist, ob sie der Vorbereitung einer militärischen Intervention oder „nur“ als politisches Druckmittel dienen.

23 Die Brandmarkung der Regierungspartei Einiges Russland als „Partei der Diebe und Gauner“ hatte Alexander Nawalny in Umlauf gebracht.

24 Die Entwicklung der Beziehungen zwischen Russland und dem Westen von einer (im Westen, insbesondere in Deutschland und der EU gehegten Illusion) „Partnerschaft“ (sogar einer „Strategischen Partnerschaft") hin zur (vom Kreml realiter aufgebauten und verfolgten) strategischen Gegnerschaft wird ausführlich behandelt bei Adomeit 2021.

25 So Bundeskanzlerin Angela Merkel am 13. Mai 2020 im Bundestag, Deutscher Bundestag - 19. Wahlperiode - 159. Sitzung, Stenografischer Bericht, S. 19700, 13.5.2020.

26 Idid. 
Zum radikal veränderten strategischen Umfeld gehört außerdem die 2011 gegründete russisch-chinesische Umfassende Strategische Partnerschaft, die nicht bloß eine militärstrategische Komponente hat und in mehreren gemeinsamen Manövern von Land-, See-, Luft- und sogar strategischen Streitkräften ihren Ausdruck findet. ${ }^{27} \mathrm{Sie}$ verfügt auch über eine gemeinsame ideologische Basis, gekennzeichnet von Autoritarismus mit anti-demokratischen, anti-pluralistischen und anti-liberalen Inhalten. ${ }^{28}$ Jedwedes strategische NATO-Konzept muss, will es richtungsweisend sein, diesen profunden Veränderungen Rechnung tragen.

\section{Was sollte das Strategische Konzept 2022 enthalten?}

Eine Grundlage für das zu erarbeitende Konzept hat der im November 2020 vorgelegte Abschlussbericht der Reflexionsgruppe geschaffen. ${ }^{29} \mathrm{Er}$ analysiert die Tendenzen, die das NATO-Umfeld von heute bis 2030 prägen, und formuliert Vorschläge für Reaktionen der NATO. Das Dokument ist mit 72 Seiten recht umfangreich und detailliert. Diagnose und Handlungsempfehlungen betreffen ganz unterschiedliche funktionale Bereiche und Regionen. Hier sollen nur die Kernaussagen zum gegenwärtigen und künftigen Verhältnis zu Russland aufgezeigt werden.

Die Charakterisierung des Russland betreffenden strategischen Umfelds unterscheidet sich in ihrer Klar-

27 Adomeit 2022.

28 Das enge russisch-chinesische Verhältnis mit der Begrifflichkeit „Partnerschaft“ wurde nicht, wie westliche Putin- und Russland-Versteher behaupten, 2014 als Reaktion auf westliche Sanktionen gegen Russland gegründet, sondern geht auf das Jahr 1994 zurück. Zwei Jahre später wurde die (einfache) Strategische Partnerschaft zur Konstruktiven Strategischen Partnerschaft und 2011 zur Umfassenden Strategischen Partnerschaft. Siehe Adomeit 2022. Die letzte Demonstration russisch-chinesischer militärischer Zusammenarbeit erfolgte im September 2021 in der Zapad-2021 Großübung.

29 Auf deutsche Initiative hatten die Staats- und Regierungschefs der NATO auf ihrem Treffen im Dezember 2019 in London Generalsekretär Jens Stoltenberg gebeten, einen zukunftsorientierten Reflexionsprozess einzuleiten, um Stärkungsmöglichkeiten der politischen Dimension des Nordatlantischen Bündnisses zu prüfen. $\mathrm{Zu}$ diesem Zweck ernannte Generalsekretär Stoltenberg im April 2020 eine unabhängige Reflexionsgruppe unter dem gemeinsamen Vorsitz von Thomas de Maizière und $\mathrm{A}$. Wess Mitchell. Mitglieder der Gruppe waren John Bew, Greta Bossenmaier, Anja Dalgaard-Nielsen, Marta Dassù, Anna Fotyga, Tacan Ildem, Hubert Védrine und Herna Verhagen. Der Abschlussbericht soll als eine der Grundlagen sowohl für das Strategische Konzept 2022 als auch für die ambitionierte Agenda „NATO 2030“ dienen. heit und Deutlichkeit radikal von früheren Strategischen Konzepten. Der Bericht stellt fest: „Nach dem Ende des Kalten Krieges hat die NATO den Versuch unternommen, auf der Grundlage des Dialogs und der praktischen $\mathrm{Zu}$ sammenarbeit in Bereichen gemeinsamer Interessen eine umfassende Partnerschaft mit Russland aufzubauen. Jedoch haben das aggressive Verhalten Russlands gegenüber Georgien und der Ukraine, gefolgt von seiner umfangreichen militärischen Aufrüstung und einem selbstbewussten Auftreten im Ostsee- und Schwarzmeerraum, im östlichen Mittelmeer und im Hohen Norden zu einer erheblichen Verschlechterung der Beziehungen geführt und sich negativ auf die Sicherheit des euro-atlantischen Raumes ausgewirkt. Russland führt regelmäßig militärische Einschüchterungsoperationen in unmittelbarer Nähe zur NATO durch und hat seine Reichweite sowie seine Fähigkeiten gesteigert, sodass es den Luftraum und die freie Schifffahrt im Atlantik bedrohen kann. Es hat zahlreiche wichtige internationale Verpflichtungen verletzt und eine Reihe konventioneller und nicht konventioneller Kapazitäten aufgebaut, die sowohl die Sicherheit einzelner NATO-Verbündeter als auch die Stabilität und Geschlossenheit des Bündnisses als Ganzes bedrohen. Russland hat hinreichend bewiesen, dass es fähig und bereit ist, mit militärischer Gewalt vorzugehen, und versucht nach wie vor, die Risse zwischen den Verbündeten sowie innerhalb der NATO-Gesellschaften auszunutzen. Außerdem hat es im Bündnisgebiet chemische Waffen verwendet, was zivile Opfer gefordert hat. [...] Es ist in der Lage, die NATO vor vollendete Tatsachen zu stellen bzw. in Krisensituationen dauerhaften und lähmenden Druck auszuüben. [...] Mit Blick auf 2030 wird Russland für das Bündnis höchstwahrscheinlich die wichtigste militärische Bedrohung bleiben.“30

Davon ausgehend empfiehlt der Abschlussbericht unter anderem: Die NATO sollte ,ihren zweigleisigen Ansatz aus Abschreckung und Dialog im Rahmen der bei den Gipfeltreffen in Wales und Warschau festgelegten Parameter fortführen." Die insbesondere in der Diskussion in Deutschland weithin und mit Nachdruck erhobene Forderung nach Intensivierung des „Dialogs“ als eine Art Zauberformel wird relativiert mit den Worten, dass „Russlands anhaltende Maßnahmen der Selbstbehauptung und sein aggressives Handeln - darunter eine hybride Kampagne zur Untergrabung des Vertrauens in die demokratischen Institutionen des Bündnisses - sich bis heute als hartnäckige Hindernisse für einen substanziellen Dialog erwiesen haben. [...] Dialog kann weder die notwendige

30 Reflexionsgruppe 2020, 25. 
Transparenz noch die Erfüllung von Verpflichtungen ersetzen, die Russland nach dem Völkerrecht oder bilateralen Abkommen eingegangen ist, einschließlich des Verzichts auf die Anwendung von Gewalt. [...] Das Bündnis sollte den NATO-Russland-Rat weiter als Hauptplattform zur Übermittlung politischer Botschaften an Russland nutzen. Der NRR sollte als Plattform dienen, um Russland gemeinsame Botschaften mit zweierlei Inhalt zu übermitteln: einerseits hinsichtlich der vertrauens- und sicherheitsbildenden Maßnahmen, andererseits zur Betonung des Bestehens des Verteidigungs- und Abschreckungsdispositivs des Bündnisses.“" ${ }^{31}$

Diese Empfehlung plädiert für die weitere Gültigkeit der im Harmel-Bericht festgelegten Doppelstrategie im Verhältnis zu Moskau - einerseits Gesprächs- und Verhandlungsbereitschaft zeigen und sich um Entspannung in den Beziehungen bemühen, andererseits Abschreckung bewirken durch ausreichende militärische Stärke, um eindeutig und zweifelsfrei die militärische Sicherheit der NATO-Mitgliedstaaten zu gewährleisten. Wie der Bericht besagt, liegt der Schwerpunkt allerdings auf Letzterem: „Die NATO muss weiterhin über angemessene konventionelle und nukleare Militärkapazitäten verfügen und die Beweglichkeit und Flexibilität besitzen, Aggressionen im gesamten Bündnisgebiet zu begegnen, auch dort wo russische Streitkräfte entweder direkt oder indirekt tätig sind, insbesondere an der Ostflanke der NATO.“32

Zudem sollte das Bündnis ,über ein dynamisches Vorgehensschema nachdenken, das es ermöglichen würde, die für Russland entstehenden Kosten einer Aggression zu steigern (beispielsweise durch koordinierte Verschärfung statt bloße Erneuerung der Sanktionen, je nachdem wie Russland sich verhält, Aufdeckung der verdeckten russischen Aktivitäten in der Ukraine usw., während gleichzeitig ein verstärktes politisches Zugehen auf Russland mit dem Ziel unterstützt werden sollte, über Maßnahmen der Rüstungskontrolle und der Risikoverringerung zu verhandeln. “"33

Besondere Bedeutung hat der obige Bezug auf die Ukraine. Auch hier ist die Botschaft des Berichts eindeutig: „Der Konflikt in der Ukraine sollte eine der wichtigsten Prioritäten auf der Tagesordnung des Nato-Russland-Rats bleiben. “34 Zudem sollte „die NATO versuchen, die Partnerschaften mit der Ukraine und Georgien auszuweiten und zu stärken, da beide verwundbare Demokratien sind, die eine Mitgliedschaft anstreben und unter ständigem

31 Reflexionsgruppe 2020, 26.

32 Reflexionsgruppe 2020, 25.

33 Reflexionsgruppe 2020, 27.

34 Reflexionsgruppe 2020, 26.
Druck von Russland stehen, der von außen und von innen ausgeübt wird.“ ${ }^{35}$ Die Reflexionsgruppe macht sich nicht ausdrücklich für Waffenlieferungen an die Ukraine stark, schließt diese allerdings nicht aus.

In ihrer letzten Empfehlung befasst sich die Gruppe mit China. „Die NATO“, so der Bericht, „sollte innerhalb der JISD [Joint Intelligence and Security Division] eine spezielle Einheit ernennen, die beobachtet und bewertet, inwiefern die russisch-chinesische Zusammenarbeit im militärischen, technologischen und politischen Bereich, einschließlich der Abstimmung bei Desinformation und hybrider Kriegsführung, die euro-atlantische Sicherheit beeinträchtigt, und dem Nordatlantikrat regelmäßig Bericht erstattet." ${ }^{\text {"36 }}$

Wie oben festgestellt, unterscheidet sich das Dokument in Ton und Inhalt scharf von den drei früheren Strategischen Konzepten. Es steht damit keineswegs allein. So forderte bereits 2017 einer von Deutschlands besten NATO-Kennern, das Bündnis müsse eine grundlegende Neubewertung seiner Position zu Russland vornehmen, denn „die Krise in Osteuropa ist kein vorübergehendes politisches Schlechtwettergebiet, sondern stellt einen grundlegenden sicherheitspolitischen Klimawandel dar. Die NATO befindet sich seit 2014 wieder in der Artikel-5Welt, in der Abschreckung und Bündnisverteidigung die primären Aufgaben des Bündnisses sind. [...] Als erste Kernaufgabe sollte in einem neuen Strategischen Konzept die Abschreckung und Verteidigung beziehungsweise der Schutz des Bündnisgebietes stehen. Anders als in der Phase nach 1991 ist dies keine rhetorische Pflichtübung mehr, sondern muss mit glaubwürdigen militärischen Fähigkeiten hinterlegt sein. Darüber hinaus sind Verteidigungspläne erforderlich, die auf verschiedene mögliche Szenarien zugeschnitten sind. ${ }^{\text {37 }}$

\section{Ausblick}

Es ist allerdings keineswegs sicher, dass das neue Strategische Konzept die Empfehlung aufgreifen wird, laut der die NATO-Staaten glaubwürdige militärische Fähigkeiten entwickeln sollten, um russische Streitkräfte etwa an der

35 Reflexionsgruppe 2020, 64. 36 Reflexionsgruppe 2020, 27.

37 Kamp 2017, 30-31. Der Autor ergänzt: „Schon seit dem Ende des Kalten Krieges wurden die öffentlichen Strategischen Konzepte um geheime militärische Zusatzdokumente ergänzt, die aus den politischen Vorgaben des Konzeptes die militärischen Folgerungen zogen. Ihnen kommt angesichts der konkreten Bedrohungen künftig eine noch größere Bedeutung zu.“ 
Ostflanke des Bündnisses abschrecken oder von ihr abhalten zu können. Insbesondere Deutschland hat an der alle drei Strategischen Konzepte durchziehenden Illusion einer „Partnerschaft“ mit Russland unbeirrt festgehalten und scheint es immer noch zu tun. Die Bundesregierung hat im Prinzip keine Doppelstrategie nach Harmel, sondern eine einseitige und eingleisige Russlandpolitik verfolgt. Deren Kern besteht letztlich darin, ein Wohlverhalten „Russlands“ (also eines Klüngels von Freunden und Weggefährten Putins sowie ehemaligen und aktiven Geheimdienstlern) durch wirtschaftliche und zivilgesellschaftliche „Verflechtung“ und Intensivierung des politischen „Dialogs“ zu erreichen. Nachdem Polen, Ungarn und Tschechien, später Estland, Lettland, Litauen, Rumänien, Bulgarien, die Slowakei und Slowenien der NATO beigetreten sind, stieß dieser Ansatz immer stärker auf Widerstand. Daher haftet NATO-Dokumenten und der auf ihnen beruhenden Politik ein Kompromisscharakter an, der den Versuch offenbart, gegenüber Moskau eine „harte“ mit einer „weichen“ Linie zu vereinen. Viele Indizien sprechen dafür, dass dieses Bemühen der Bundesregierung ungebrochen bleibt. $\mathrm{Zu}$ den Beweisstücken gehören die Wahlprogramme und Wahlkampagnen der politischen Parteien in Deutschland, in denen sicherheitspolitische Themen praktisch keine Rolle gespielt haben. Das lässt erwarten, dass das künftige Strategische Konzept der NATO der von der Reflexionsgruppe und Sicherheitsexperten konstatierten Notwendigkeit, das Verhältnis zu Russland grundlegend neu zu bestimmen, nicht folgen wird.

\section{Literatur}

Adomeit, Hannes (2017a): Innenpolitische Determinanten der Putinschen Außenpolitik, Sirius - Zeitschrift für Strategische Analysen, 1 (1), 33-52

Adomeit, Hannes (2017b): Altes Denken, statt Neues Russland: Innenpolitische Bestimmungsfaktoren der russischen Außenpolitik, Portal Politikwissenschaft, 26. September, https://www.pw-portal.de/putins-russland/40508-altesdenken-statt-neues-russland

Adomeit, Hannes (2020): Bilanz der deutschen Russland-Politik seit 1990. Sirius - Zeitschrift für strategische Analysen, 4(3), 276-292
Adomeit, Hannes (2021): Russland und der Westen: Von „strategischer Partnerschaft“ zur strategischen Gegnerschaft, Sirius: Zeitschrift für strategische Analysen, 5(2), 107-124

Adomeit, Hannes (2022): Russia's Strategic Outlook and Policies: What Role for China? In: Kirchberger, Sarah, Sinjen, Svenja und Wörmer, Nils: Russia-China Relations: Emerging Alliance or Eternal Rivals? Springer Verlag, Frühjahr 2022 (voraussichtlich) Blank, Stephen (2008): Russia versus NATO in the CIS. Prag: RFE/RL Research Report, 14.5.2008

Kamp, Karl-Heinz (2017): Grundzüge einer neuen NATO-Strategie, Sirius - Zeitschrift für strategische Analysen, 1(1), 25-33

Socor, Vladimir (2008): Moscow Makes Furious but Empty Threats to Georgia and Ukraine, Eurasia Daily Monitor, 5(70), Jamestown Foundation

\section{Offizielle Veröffentlichungen und Dokumente}

Europäischer Rat (1999): Gemeinsame Strategie der Europäischen Union vom 4. Juni 1999 für Russland (1999/414/GASP), Amtsblatt der Europäischen Gemeinschaften L 157/1, 24. Juni, https://eur-lex.europa.eu/legal-content/DE/TXT/HTML/?uri=C ELEX:31999E0414\&from $=E N$

Reflexionsgruppe (2020): NATO 2030. Geeint in ein neues Zeitalter. Analyse und Empfehlungen der vom NATO-Generalsekretär eingesetzten Reflexionsgruppe, 25.11.2020, Webseite des Auswärtigen Amtes; https://www.auswaertiges-amt.de/blob/ 2439466/0852f283a611b62ee0c852a700c4a820/201202reflexionsgruppe-ergebnisse-arbeitsuebersetzung-data.pdf Strategisches Konzept (1991): North Atlantic Treaty Organisation. The Alliance's Strategic Concept Approved by the Heads of State and Government participating in the meeting of the North Atlantic Council in Washington D.C., 7-8 November 1991, NATO Webseite; https://www.nato.int/cps/en/natohq/ official_texts_23847.htm

Strategisches Konzept (1999): North Atlantic Treaty Organisation. The Alliance's Strategic Concept Approved by the Heads of State and Government participating in the meeting of the North Atlantic Council in Washington D.C., 24 April 1999, NATO Webseite; https://www.nato.int/cps/en/natolive/official_ texts_27433.htm

Strategisches Konzept (2010): North Atlantic Treaty Organisation. Active Engagement, Modern Defence: Strategic Concept for the Defence and Security of the Members of the North Atlantic Treaty Organisation adopted by Heads of State and Government in Lisbon, 19 November 2010, NATO Webseite; https://www. nato.int/cps/en/natohq/official_texts_68580.htm 